\title{
Magnetic structure of azurite above the 1/3 magnetization plateau
}

\author{
F. Aimo, ${ }^{1}$ S. Krämer, ${ }^{1}$ M. Klanjšek, ${ }^{1,2,3}$ M. Horvatić, ${ }^{1, *}$ and C. Berthier ${ }^{1}$ \\ ${ }^{1}$ Laboratoire National des Champs Magnétiques Intenses, LNCMI - CNRS (UPR3228), \\ UJF, UPS and INSA, BP 166, 38042 Grenoble Cedex 9, France \\ ${ }^{2}$ Jožef Stefan Institute, Jamova cesta 39, 1000 Ljubljana, Slovenia \\ ${ }^{3}$ EN-FIST Centre of Excellence, Dunajska 156, 1000 Ljubljana, Slovenia
}

(Dated: November 1, 2018)

\begin{abstract}
The transition from the 1/3 magnetization plateau towards the saturation magnetization in azurite has been studied by low-temperature, high-magnetic-field, high-frequency proton nuclear magnetic resonance (NMR). The observed symmetrical splitting of the NMR spectra is incompatible with the longitudinal incommensurate order appearing when the longitudinal correlation function becomes dominant over the transverse one, which is the expected framework for the existence of the $2 / 3$ magnetization plateau. The spectra are rather interpreted in terms of a more standard transverse antiferromagnetic (canted) order.

PACS numbers: 75.10.Pq, 67.80.dk, 75.25.-j, 76.60.-k
\end{abstract}

Since the discovery of the magnetization plateau at $1 / 3$ of the saturation magnetization in the natural mineral azurite [1], $\mathrm{Cu}_{3}\left(\mathrm{CO}_{3}\right)_{2}(\mathrm{OH})_{2}$, the system has been intensely studied as a model for the frustrated antiferromagnetic Heisenberg spin- $1 / 2$ chain of "distorted diamond" geometry [2]. Its large $1 / 3$ plateau is of pure quantum origin, as it is based on the spin configuration where two more strongly coupled "dimer" spins are in a singlet state, while the third "monomer" spin is fully polarized [2, 3]. In this letter we focus on another interesting subject, the possible existence of a narrow $2 / 3$ plateau (so far unresolved by the magnetization measurements [1, 4]) in the middle of the increase of magnetization from the $1 / 3$ plateau towards the fully polarized system [2, 5, 6]. Unlike the $1 / 3$ plateau, the $2 / 3$ plateau is expected from Oshikawa's criterion [7] to break the translational symmetry. Within the one-dimensional (1D) description (i.e., the Tomonaga-Luttinger description), where the spin-spin correlation functions along the chain exhibit a power-law behavior, it is associated to a rather exceptional situation in which the longitudinal spin-spin correlation function becomes dominant over the transverse one due to the so-called " $\eta$ inversion" of the corresponding exponents $\left(\eta^{\|}<\eta^{\perp}\right)[\underline{5},[6]$. In this case an incommensurate (IC) longitudinal (i.e., parallel to the applied field) three-dimensional (3D) order is expected to be stabilized at low temperature, which can generate a plateau at the commensurate point. Theoretically it has been shown that the $2 / 3$ plateau may exist in a diamond spin chain [2, 5, 6] for certain values of exchange couplings $J_{1}, J_{2}, J_{3}$ lying within the relatively broad range of values extracted from the experiments [1, 8-13]. Namely, there is a controversy on these values, which seems to have converged only very recently [13].

Another, more conventional and thus less interesting situation appears when the transverse correlations are dominant, leading to a transverse antiferromagnetic (AF) order at low temperature, meaning that the dimer spins develop a canted polarization. The order would typically be of the Néel type where the direction of the transverse polarization is fixed, but it may also be a spiral IC order where the transverse polarization rotates along the chain. In this work we present nuclear magnetic resonance (NMR) data which are clearly in favor of the Néel canted order. We thus exclude the more appealing possibility of the longitudinal IC order leading to a $2 / 3$ plateau.

In order to determine the microscopic nature of the spin structure of azurite above the $1 / 3$ plateau, we performed a high-frequency $(1.3-1.45 \mathrm{GHz})$ proton NMR at very high field (31-34 T) in the M9 magnet at LNCMIGrenoble, using specially designed ("bottom tuned") NMR probe for the narrow bore ${ }^{3} \mathrm{He}$ refrigerator. We mention that, in contrast to the previous NMR work at lower field [3], the spin structure in this field range cannot be directly accessed by the copper NMR, because the signal is lost ("wiped out" by shortening of $T_{2}$ ) due to enhanced fluctuations of electronic spins. There is only one proton site in azurite, which however generates two NMR lines for an arbitrary direction of the magnetic field $H$, corresponding to two diamond chains per unit cell which are differently oriented with respect to $H$ (see Fig. 1 in Ref. 3]). When the field is applied within the $a c$-plane of mirror symmetry, both chains become equivalent and only one proton NMR line is observed. In this study the azurite single crystal was oriented with its $c$-axis approximately parallel to the magnetic field, so that the field is perpendicular to the chain $b$-axis, and only one proton NMR line is expected for a homogeneous system.

Indeed, in the field dependence of proton NMR spectra shown in Fig. 1 a single line is observed in the 1/3 plateau, that is below the second critical field $H_{\mathrm{c} 2}(0.6 \mathrm{~K})=$ $31.35 \mathrm{~T}$. The "activated" magnetic field dependence of the nuclear spin-lattice relaxation rate data $\left(T_{1}^{-1}\right.$, not shown here) confirms that this field range corresponds to a linear closing of the gap at the end of the plateau, as is 


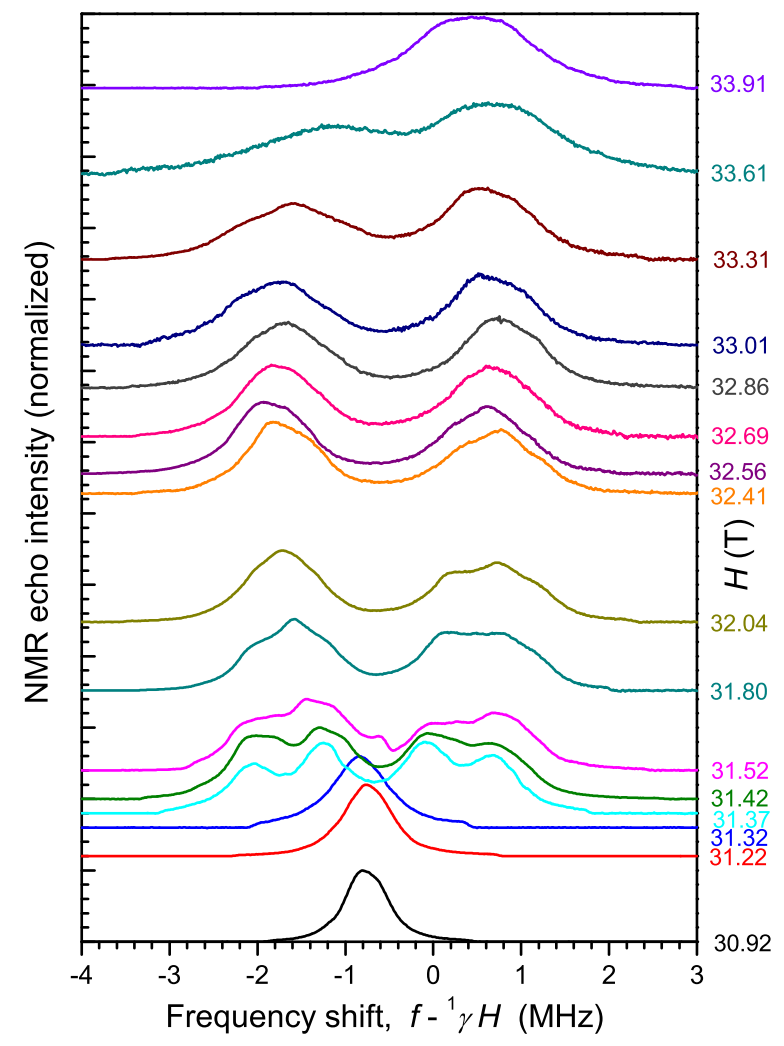

FIG. 1: (color online) High-field proton NMR spectra of azurite covering the transition from the $1 / 3$ plateau into the fully polarized system, taken at $0.6 \mathrm{~K}$. Vertical offset of the spectra is proportional to the magnetic field, applied parallel to the $c$-axis. Frequency axis is taken relative to the proton Larmor frequency defined by its gyromagnetic ratio, ${ }^{1} \gamma=$ $42.5774 \mathrm{MHz} / \mathrm{T}$.

also observed by high-field EPR [14]. At $H_{\mathrm{c} 2}$ the NMR line suddenly broadens and then splits into a nearly symmetrical spectrum. Each side of the spectrum consists of a pair of lines just above $H_{\mathrm{c} 2}$. Each pair merges into a distribution with some structure, and transforms into a more regular Gaussian lineshape at higher fields.

As the spectrum is nowhere completely split, it is difficult to directly distinguish if the lineshape corresponds to a simple Néel type splitting into two (or $2 \times 2$ ) somewhat overlapping lines centered at two frequencies $f_{1}$ and $f_{2}$, or to an IC lineshape. In the latter case, for a $1 \mathrm{D}$ sinusoidal distribution of frequencies spreading between $f_{1}$ and $f_{2}$, an NMR lineshape $I(f)$ corresponds to the density function $D(f) \propto\left[\left(f-f_{1}\right)\left(f_{2}-f\right)\right]^{-1 / 2}$ convoluted by some line-broadening function $G(f), I(f)=D * G(f)$. In Fig. 2 we have performed the corresponding fits to two selected spectra presenting the most regular lineshape, one just above $H_{\mathrm{c} 2}$ and the other in the middle of the transition towards the saturation. In the former case the best fit was achieved using ad hoc Lorentzian line-broadening function, while the latter spectra were better approximated by a Gaussian line-broadening. In both cases the fits are in favor of an AF ordered system, while fits to the lineshape corresponding to an IC state clearly fail.

Further quantitative analysis of the spectra is given in Fig. 3. We first observe that the first moment of the complete lineshape, which reflects an average spin polarization in the system, is barely changing although the magnetization of the system strongly increases. This can be attributed to a nearly zero value of the longitudinal coupling constant $\mathrm{A}_{z z}$ of protons for this particular orientation of the sample, due to the cancellation of the hyperfine (super-exchange induced) and the direct dipolar component.

In order to quantify the splitting of the spectra shown in Fig. 1, which is apparently nearly field independent, we have fitted the spectra to two Gaussians (see Fig. 2) and plotted in Fig. 3 their position taken with respect to the $1^{\text {st }}$ moment of each spectrum. For the three spectra close to $H_{\mathrm{c} 2}$, where the fit to $2 \times 2$ peaks is more appropriate, the splitting is defined from the position of the weighted average of the two peaks on each side. In this way we find that the splitting of the spectra is highly symmetrical and nearly flat with very weakly expressed maximum at $H_{2 / 3}=32.6 \mathrm{~T}$, close to the midpoint between $1 / 3$ magnetization and saturated magnetization. It turns out that the observed field dependence of the line splitting follows precisely the prediction (see Fig. 3) for the transverse staggered spin polarization $S^{\perp}$ in the simplest model of independent dimers, which has originally been employed to describe the Bose-Einstein condensation (BEC) of triplets [15, 16]. We observe that close to $H_{\mathrm{c} 2}$ one of the Zeeman-split triplet states of a dimer becomes degenerate with the singlet. Mixing these two states produces a state where the two spins of a dimer acquire identical longitudinal polarizations $S^{z}$ and opposite transverse polarizations $S^{\perp}$, which depend on the mixing coefficients [15]. The magnitudes of the two components

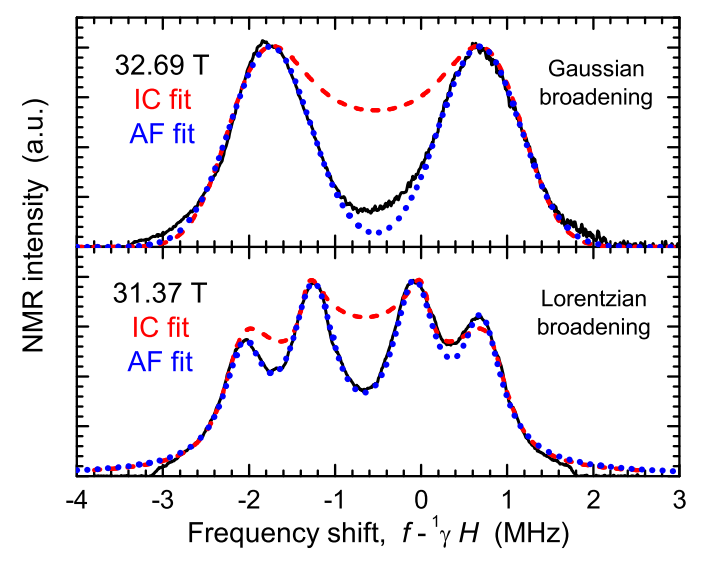

FIG. 2: (color online) Fits of two representative proton NMR spectra (solid lines) to the lineshapes corresponding to an AF ordered state (dotted lines) and to a 1D IC state (dashed lines). 


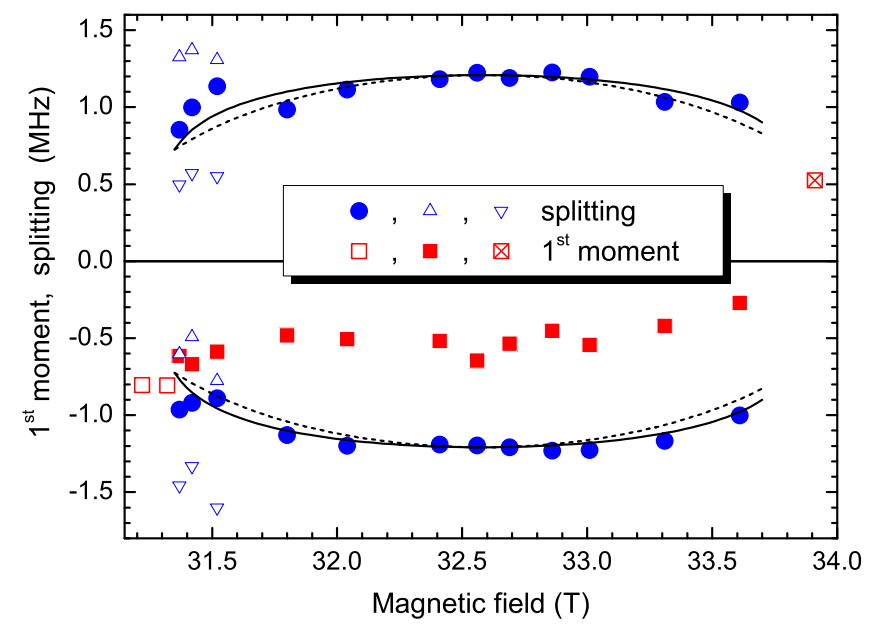

FIG. 3: (color online) Analysis of the spectra given in Fig. 1 The first moment, i.e. the average lineshift with respect to the Larmor frequency, in the $1 / 3$ plateau ( $\square$ ), in the transition region $(\boldsymbol{\square})$, and close to full polarization $(\otimes)$. The position of two $(\bullet)$ or four $(\triangle, \nabla)$ components of the nearly symmetrically split spectra determined relative to the 1 st moment. Close to the $1 / 3$ plateau, where $2 \times 2$ components fit is applied, - denotes the weighted average position of $\Delta$ and $\nabla$. Solid and dashed lines are predicted field dependence for 1D and $3 \mathrm{D}$ system of weakly interacting dimers, respectively (see the text).

are related by an elliptical dependence:

$$
S^{\perp}=\sqrt{2} \sqrt{1 / 4^{2}-\left(S^{z}-1 / 4\right)^{2}}
$$

where $S^{\perp}$ has a broad maximum at the point where the spins are half polarized, $S_{\max }^{\perp}=S^{\perp}\left(S^{z}=1 / 4\right)=\sqrt{2} / 4$, and develops very rapidly from zero, $S^{\perp}\left(S^{z}=0,1 / 2\right)=$ 0 , as soon as the system departs from zero or full polarization. In azurite the polarization of dimers in the $1 / 3$ plateau is found to be $10 \%$ [3], so that we expect that at $H_{\mathrm{c} 2}$ the transverse polarization will develop starting right away from as much as $S^{\perp}\left(S^{z}=1 / 20\right)=0.6 S_{\max }^{\perp}$. Eq. 11 can be used to predict the field dependence of $S^{\perp}$, if $S^{z}(H)$ is known from the experiment or from the theory. High field magnetization data in azurite [4] show that the increase of magnetization towards saturation is of the "arcsin" type corresponding to what is expected for a 1D system. Our 1D fit shown in Fig. 3 corresponds to the arcsin dependence defined by the initial point $S^{z}\left(H_{\mathrm{c} 2}\right)=$ $1 / 20$ and the midpoint $S^{z}\left(H_{2 / 3}\right)=1 / 4$. For comparison, we also show a $3 \mathrm{D}$ fit, where the $S^{z}(H)$ dependence is taken to be linear, passing through the same points. The 1D fit is excellent, in particular when we take into account that the only fitting parameters are the overall scaling defined by the observed maximum splitting of $\Delta f_{\max }=1.2 \mathrm{MHz}$ and the corresponding field value $H_{2 / 3}$. The $3 \mathrm{D}$ fit is very close, and the experimental data do not really allow us to distinguish between the two fits. The fits confirm the description in terms of the transverse polarization, whose main characteristics is that strong increase of magnetization is accompanied by nearly field independent splitting of the spectra. We note that this particular type of behavior is not expected in a description in terms of an IC modulation of the longitudinal polarization, which provides another argument against this latter interpretation.

In the following we proceed by a quantitative analysis of the experimental value $\left(\Delta f_{\max }\right)$ of the splitting. A transverse component of the spin polarization is shifting the NMR line through the off-diagonal $\left(\mathrm{A}_{z x}\right)$ elements of the coupling tensor. In general both the direct dipolar field, as well as the hyperfine field are expected to contribute to the proton coupling tensor. However, the latter mechanism is based on an $s$-type wave function at the proton site, it is thus isotropic and cannot generate $\mathrm{A}_{z x}$ elements. This means that the NMR splitting due to the transverse staggered $S^{\perp}$ is of pure dipolar origin and can be calculated for a given spin order from the known crystallographic structure [17 19]. In Fig. 4 we show so predicted NMR linesplitting generated by $\pm S_{\max }^{\perp}= \pm \sqrt{2} / 4$ on dimer spins as a function of their direction in the plane perpendicular to the field applied along the $c$-axis. There are two possibilities for the stacking of $S^{\perp}$ (AF1 [20] and $\mathrm{AF} 2)$ as regards the relative phase $(0$ or $\pi)$ of an AF spin arrangement in two chains in a unit cell. Comparing the prediction to the experimental value of $\pm 1.2 \mathrm{MHz}$, we observe that this is very close to the predicted $\pm 1.35 \mathrm{MHz}$ for the AF1 type order with $S^{\perp}$ along the $a^{*} \cong a$-axis. This particular direction agrees with the easy magnetization axis (perpendicular to the chains), as defined by the low-field magnetization measurements [1]. However, the magnetization data correspond to the initial polarization of the monomer spins, while in the high-field measurement these spins are saturated and thus inert, and the

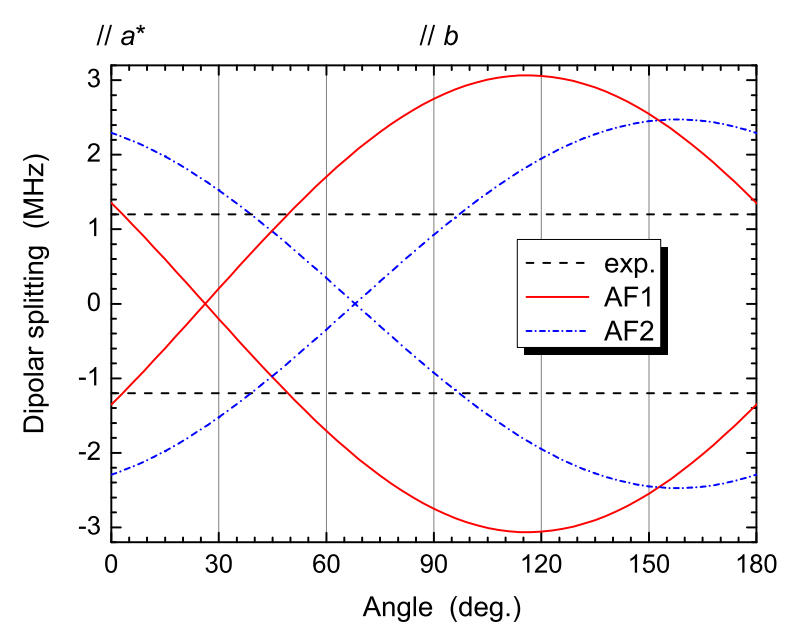

FIG. 4: (color online) Experimental value of the line splitting (dashed line) compared to the predicted dipolar splitting for two possible AF orders (solid and dash-dotted line, as a function of the orientation of the ordered moments in the transverse plane. 
active sites are dimers. Unfortunately, there is no direct information on the "local" easy axis of dimers at high field. In general, azurite presents quite strong anisotropy of its magnetic properties, which has not yet been understood [1, 6, 8, 18.

One should remark that the predicted splitting from Eq. 1 corresponds to the maximum expected $S^{\perp}$, which is then to some extent reduced by thermal and quantum fluctuations. According to the complete mean field solution for the 3D interacting dimers [21], the thermal effects are negligible below $0.4 T_{c}^{\max }$, where $T_{c}^{\max }$ is the maximum critical temperature. From measurements taken at $1.2 \mathrm{~K}$ we know that $T_{c}^{\max }$ is well above this temperature, meaning that the low temperature limit is fulfilled for our $0.6 \mathrm{~K}$ spectra. The effect of quantum fluctuations depends strongly on dimensionality and can be very important for purely $1 \mathrm{D}$ systems. For example, in the $\mathrm{CuBr}_{4}\left(\mathrm{C}_{5} \mathrm{H}_{12} \mathrm{~N}\right)_{2}(\mathrm{BPCB})$ spin ladder, where the effective $3 \mathrm{D}$ coupling is $\approx 50$ times smaller than the inter-dimer interactions, the reduction of the transverse moment is $\approx 50 \%$ [22, 23]. In azurite the 3D exchange couplings [13] are estimated to be of the order of a few $\mathrm{K}$, that is (only) one order of magnitude smaller than the (effective) 1D couplings. At high magnetic field the 1D Hamiltonian of azurite can be further reduced to the effective XXZ, $S=1 / 2$ spin chain Hamiltonian describing only the dimer spins 24, 25]. The corresponding couplings are of the order of $1 \mathrm{~K}$, of the same size as the ordering temperature, so that we can suspect that residual $3 \mathrm{D}$ couplings are of comparable size. The 3D character of azurite at high field is thus probably important, making the effect of quantum fluctuations relatively weak. For example, comparing our prediction for $S^{\perp}$ along the $a^{*}$-axis to the experimental value we get the reduction of only $\approx 10 \%$. We also recall that this description does not take into account the anisotropy terms of azurite's Hamiltonian [1, 6, 8, 18], which might also significantly modify the observed spin polarizations.

Finally, within the effective XXZ Hamiltonian the anisotropy of the numerically optimized [25] effective coupling is found to be $J_{x y} / J_{z}=2.2$, very close to the value of 2 obtained when the same description is applied to the spin ladder in the strong coupling regime [23]. For this latter system we know that in the $3 \mathrm{D}$ ordered phase the spins are canted and that no plateau is expected, in agreement with our NMR results.

In conclusion, the high-field proton NMR spectra of azurite have been analyzed to determine which type of spin order is realized between the $1 / 3$ magnetization plateau and the full polarization of the system. Both the observed line shape and the field dependence of the splitting of the spectra clearly correspond to the "conventional" transverse (canted) Néel order. They are both incompatible with the longitudinal IC order, meaning that there is no $2 / 3$ plateau in azurite, and that the corresponding "more interesting" physics does not apply to this system. These conclusions are supported by the recent theoretical estimates of the effective Hamiltonian for this system [25].

We acknowledge fruitful discussions with B. Grenier, A. Honecker, H. Ohta, and T. Sakai. Part of this work has been supported by the French ANR project NEMSICOM and by European Commission through the Transnational Access Specific Support Action (Contract No. RITA-CT2003-505474), by the EuroMagNET network (Contract No. RII3-CT-2004-506239), and by the ARRS project No. J1-2118.

* Email address: mladen.horvatic@lncmi.cnrs.fr

[1] H. Kikuchi et al., Phys. Rev. Lett. 94, 227201 (2005).

[2] K. Okamoto, T. Tonegawa, and M. Kaburagi, J. Phys. Condens. Matter 15, 5979 (2003).

[3] F. Aimo et al., Phys. Rev. Lett. 102, 127205 (2009).

[4] H. Kikuchi et al., Progr. Theor. Phys. Suppl. 159, 1 (2005).

[5] T. Sakai, K. Okamoto, and T. Tonegawa, J. Phys.: Conf. Ser. 145, 012065 (2009).

[6] T. Sakai, K. Okamoto, and T. Tonegawa, J. Phys.: Conf. Ser. 200, 022052 (2010).

[7] M. Oshikawa, M. Yamanaka, and I. Affleck, Phys. Rev. Lett. 78, 1984 (1997).

[8] B. Gu and G. Su, Phys. Rev. B 75, 174437 (2007).

[9] B. Gu and G. Su, Phys. Rev. Lett. 97, 089701 (2006); H. Kikuchi et al., Phys. Rev. Lett. 97, 089702 (2006).

[10] K. C. Rule et al., Phys. Rev. Lett. 100, 117202 (2008).

[11] H.-J. Mikeska and C. Luckmann, Phys. Rev. B 77, 054405 (2008).

[12] J. Kang et al., J. Phys.: Condens. Matter 21, 392201 (2009).

[13] H. Jeschke et al., preprint, arXiv:1012.1090

[14] H. Ohta et al., J. Phys. Soc. Jpn. 72, 2464 (2003).

[15] T. Momoi and K. Totsuka, Phys. Rev. B 62, 15067 (2000).

[16] A BEC cannot apply to azurite because of its magnetic anisotropy (discussed later on).

[17] E. L. Belokoneva et al., Phys. Chem. Miner. 28, 498 (2001); B. Grenier, unpublished data, private communication.

[18] K. C. Rule et al., Phys. Rev. B 83, 104401 (2011).

[19] The longitudinal spin polarization does not need to be taken into account because it is homogeneous over all the dimer spins and therefore cannot produce any linesplitting.

[20] In AF1 the dimers related by the $(0,1 / 2,-1 / 2)$ translation vector have the same spin polarization, as in Fig. 6 of Ref. [18].

[21] M. Tachiki and T. Yamada, J. Phys. Soc. Jpn. 28, 1413 (1970).

[22] M. Klanjšek et al., Phys. Rev. Lett. 101, 137207 (2008).

[23] P. Bouillot et al., Phys. Rev. B 83, 054407 (2011).

[24] A. Honecker and A. Läuchli, Phys. Rev. B 63, 174407 (2001).

[25] A. Honecker et al., J. Phys.: Condens. Matter, accepted for publication, preprint, arXiv:1103.xxxx. 